How to cite this article:

Nasir, H. J. A., Ku-Mahamud, K. R., \& Kamioka, E. (2019). Parameter adaptation for ant colony system in wireless sensor network. Journal of Information and Communication Technology, 18(2), 167-182.

\title{
PARAMETER ADAPTATION FOR ANT COLONY SYSTEM IN WIRELESS SENSOR NETWORK
}

\author{
${ }^{1}$ Husna Jamal Abdul Nasir, ${ }^{2}$ Ku Ruhana Ku-Mahamud \& \\ ${ }^{3}$ Eiji Kamioka \\ ${ }^{1}$ Universiti Malaysia Perlis, Malaysia \\ ${ }^{2}$ Universiti Utara Malaysia, Malaysia, \\ ${ }^{3}$ Shibaura Institute of Technology, Japan \\ husna.jamanas@gmail.com;ruhana@uum.edu.my; \\ kamioka@shibaurait.ac.p
}

\begin{abstract}
The Ant Colony System (ACS) algorithm has been applied in solving packet routing problems in Wireless Sensor Networks (WSNs). Solving these problems is complicated as packets need to be submitted through sensor nodes which are spatially distributed and heterogeneous by nature. Without an effective packet routing algorithm, energy consumption will be increased while network lifetime will be reduced. Most researches are focused on optimizing the routing process by using predefined parameters within a certain range. However, this approach will not guarantee optimal performance. This paper presents the parameter adaptation values for ACS experimental set-up in validating its performance. Possible values of each parameter within a defined range were employed. Experiments were conducted to obtain the best value of each parameter to be used for throughput, energy consumption, and latency. Results of this study can be adopted to achieve optimal performance for the packet routing process.
\end{abstract}

Keywords: Ant colony optimization, parameter tuning, performance evaluation. 


\section{INTRODUCTION}

A Wireless Sensor Network (WSN) consists of tiny-sized sensor nodes that can communicate between each other, perform basic computation operations, and sense any changes in a system (Cecílio \& Furtado, 2014). Sensor nodes that are geographically distributed in the WSN are responsible for transmitting packets from source node to destination node but have limited capabilities such as memory, storage, communication capabilities, and battery power. Packets are sent using a multi-hop technique due to the limited transmission range of each sensor node. WSNs have been successfully applied in many real applications such as environmental monitoring (Ferdoush \& Li, 2014), healthcare (Tennina et al., 2014), military (Ismail, Shukran, Isa, Adib, \& Zakaria, 2018), and industrial applications (Sandra et al., 2017).

Many researchers have applied ant based routing algorithms to route packets from source node to destination node such as the Ant System (AS) by Camilo, Carreto, Silva, and Boavida (2006) and Max-Min Ant System by Fidanova and Marinov (2014). Performance metrics commonly used in evaluating the performance of routing algorithms include delay, throughput, packet loss rate, energy efficiency, energy consumption, and network lifetime. Standard values for parameters, applied by Stützle et al. (2011), in solving the travelling salesman problem in general have been adopted by researchers. However, there is no research that focuses on parameter adaptation to be used in experimental set-ups even though it has been noted that different application domains have certain dependencies in which their parameters cannot be fully adopted in other application domains (Wong, 2008).

This paper presents the analysis of parameter adaptation that can be used by ACS in WSN. ACS is a variant of the Ant Colony Optimization (ACO) algorithms and it is an improvement of the AS algorithm. ACS uses a heuristic function to construct routing solutions in dynamically-distributed environments. It consists of three main phases: solution construction, local pheromone and global pheromone updates that are influenced by the values of the parameters. Section 2 presents previous works based on ACO in WSNs while Section 3 describes the parameter adaptation. Section 4 discusses the experimental results whereas Section 5 focuses on concluding remarks and future work.

\section{ACO WORKS IN WIRELESS SENSOR NETWORKS}

The ACO algorithm is inspired by the foraging behavior of ants in finding the shortest path from nest to food source (Blum, 2005). Pheromone is a 
chemical substance that is used as a communication medium between ants in a colony which can mark a selected path during foraging behavior. Each ant will deposit pheromone during its movement to a food source and vice versa (Dorigo \& Stützle, 2004). The shortest path or optimal path can be sensed by other follower ants based on the pheromone value released by previous ants. Longer paths are indicated by lower pheromone values while paths with higher pheromone values indicate shorter paths. This cooperative behavior and other unique features prove that ACO is suitable in building a new algorithm. Many NP-complete problems such as the travelling salesman problem (Brezina \& Čičková, 2011), fault tolerance (Bukhari, Ku-Mahamud, \& Morino, 2017), sequential ordering (Skinderowicz, 2015), grid scheduling (Ku-Mahamud, Din, \& Nasir, 2011), and data classification (Al-Behadili, KuMahamud, \& Sagban, 2018) have been solved using ACO algorithms. ACO has also been applied to solve routing problems in WSNs because it is suitable to be implemented in static, mobile, and dynamic WSN environments.

The Energy-Efficient Ant-Based Routing (EEABR) algorithm was proposed by Camilo et al. (2006) to minimize energy consumption and communication load in WSNs. EEABR uses two types of ants, the forward ant that finds the high capacity sensor nodes during the search process and the backward ant that is responsible for updating the pheromone value on the sensor nodes along the path that leads to the destination node. The capacity of each neighbor node is evaluated by the forward ant based on the probabilistic decision rule. On the other hand, the global pheromone update is applied by the backward ant to encourage the ant in the next iteration to select the optimal sensor nodes. The energy efficiency of the EEABR algorithm has been evaluated under three different conditions: static network, mesh network, and mobile network. From the experimental results, the EEABR algorithm showed the highest energy efficiency when compared with the other two ant-based routing algorithms: Basic Ant-Based Routing (BABR) and Improved Ant-Based Routing (IABR). However, the exploration to an alternative path has not been considered by the EEABR algorithm that could lead to hotspot problems where certain sensor nodes would be heavily utilized as compared to other available sensor nodes.

Rao and Rani (2015) proposed a hybrid routing algorithm that combines ACO and the cluster technique to increase the energy efficiency and network lifetime of a WSN system. Sensor nodes are grouped into clusters and the best sensor node in terms of its distance to destination node and residual energy is selected as a cluster head in the cluster. During packets submission to the destination node, each cluster member will send packets to the cluster head 
to be forwarded to the destination node. ACO is responsible for finding the optimal path between the cluster head and the destination node where the pheromone update technique is executed on a selected path to overcome stagnation problems in the WSN. Experiments were performed to compare the performance of the proposed algorithm with LEACH and PARA algorithms. The proposed algorithm showed better results in terms of the number of survival nodes and energy consumption when compared with others. Nevertheless, other important performance metrics such as throughput, delay, and load balancing were not considered. Without effective load balancing, hotspot problems may occur in the WSN which will eventually lead to decrease in throughput and increase in delay.

The Smart Routing Algorithm (SRA) proposed by Bouarafa, Saadane, and Rahmani (2018) aims to improve the routing performance in the WSN and prolong the network lifetime of the system. The predecessor node will broadcast the request message to all successors, which are neighbor nodes, during the searching process to the destination node. The predecessor's ID will be stored in the neighbor list once it is received by its successors. At the same time, successors will broadcast their ID, location, and remaining energy to corresponding predecessor nodes. These processes are established to connect the receiver and sender to each other for communication. The SRA calculates the probability of each node based on the acknowledgments obtained from successors. The remaining energy and distance between two nodes are part of the elements used to calculate the probability value. Pheromone update, that considers the path length and evaporation rate value, will be applied to selected sensor nodes once a packet has successfully arrived at the destination node. The performance of the SRA in terms of energy consumption and path length was evaluated by a set of experiments. There were no dead nodes during the experiments undertaken in 50 iterations of the SRA. This proves that the SRA can preserve a network's lifetime by balancing the load and remaining energy among available sensor nodes. Despite the promising results of the SRA, its performance has not been evaluated against other algorithms.

Based on the recent research, it can be seen that ACO is very promising to improve the routing performance of a WSN. However, apart from the range, none of the previous research specifically defines the value of each parameter. The objective of the current research is to identify, specifically, the optimal value of each parameter that can be adopted by an ACS in WSN packet routing. 


\section{ANT COLONY SYSTEM ALGORITHMS FOR WIRELESS SENSOR NETWORKS}

The ACS was proposed by Dorigo and Gambardella (1997a, 1997b) to improve the performance of the AS. The ACS uses the same foraging behavior concept as in the AS but differs in three main aspects. The ACS uses a more aggressive action choice rule where the pheromone is only added to the global best solution, and some pheromone will be removed from each visited path. The ACS applies three main phases: solution construction, local pheromone update, and global pheromone update (Skinderowicz, 2017). The solution construction will be initialized during the movement of an ant from node to node. This is based on the pseudorandom proportional rule that exploits the previous solution and probability distribution that explores the new potential solution as applied in the AS. The pseudorandom proportional rule, also known as state transition rules, will determine the best sensor node with the highest energy level and highest pheromone value. When forward ant $k$ moves from one sensor node to another, it will select the node based on the pseudorandom proportional rule calculated as in (1):

$$
P_{(r, s)}^{k}=\left\{\begin{array}{lrr}
\operatorname{argmax}\left\{\left[\tau_{(r, s)}\right]\left[E_{v}\right]^{\beta}\right\} & \text { if } q \leq q_{0} & \text { (exploitation) } \\
S & \text { otherwise } & \text { (exploration) }
\end{array}\right.
$$

Where $P_{(r, s)}^{k}$ is the probability value of ant $k$ to move from node $r$ to node $s$ which is based on the pheromone value and heuristic value. $\tau_{(r, s)}$ is the pheromone value of the edge between node $r$ and node $s$ while $E_{v}$ is the heuristic value given by $\frac{1}{\mathrm{E}_{\mathrm{r}}}$ where $E_{r}$ is the residual energy of node $s$. The heuristic value in the pseudorandom proportional rule is controlled by the important parameter $\beta$. Based on the research by Gaertner and Clark (2005), the ideal value of $\beta$ is more than 0 . On the other hand, the possibility to explore or exploit is based on the $q$ value ranging from 0 to $1, q_{o}\left(0 \leq q_{o} \leq 1\right) . S$ is a random variable based on the probabilistic decision rule as in (2):

$$
S_{(r, s)}^{k}=\frac{\left[\tau_{(r, s)}\right]\left[E_{v}\right]^{\beta}}{\sum\left[\tau_{(r, s)}\right]\left[E_{v}\right]^{\beta}}
$$

The ACS applies two types of pheromone update techniques which are local pheromone update and global pheromone update (Gilmour \& Dras, 2005). Local pheromone update is applied to all visited sensor nodes during path construction while global pheromone update is only applied by the best ant 
after all ants have finished constructing a path and the destination node is discovered. In order to reduce the attractiveness of the visited sensor node, the local pheromone update is applied with the aim to encourage exploration to other potential sensor nodes while balancing the load in the system. The local pheromone update is calculated by (3):

$$
\tau_{(r, s)}=(1-\xi) *\left(\tau_{(r, s)}\right)+\xi\left(E_{i-} E_{r)}\right.
$$

where $\tau_{(r, s)}$ is the current pheromone value of the node and $\xi$ is the parameter value $(0 \leq \xi \leq 1)$ that can control the pheromone values. $E_{i}$ is the initial energy of the sensor node while $E_{r}$ is the residual energy of the sensor node. The forward ant is transformed to the backward ant once it arrives at the destination node. The global pheromone update will be applied by the backward ant to increase the pheromone value of the selected path. This approach will increase the attractiveness of optimal sensor nodes to the ant in the next iteration. The global pheromone update is adopted from Dorigo and Stützle (2004) and defined by (4):

$$
\tau_{(r, s)}=(1-\rho) * \tau_{(r, s)}+\left(\Delta \tau_{(r, s)}\right)
$$

where $\rho(0<\rho<1)$ is the evaporation rate value and $\Delta \tau_{(r, s)}$ is calculated by (5):

$$
\Delta \tau_{(r, s)}=\frac{1}{N_{r}}
$$

where $N_{r}$ is the number of visited nodes from node $r$ to the destination node.

\section{EXPERIMENTAL RESULTS}

Experiments were conducted to discover the best parameter to be used by an ACS in a WSN. The objective of these experiments was to discover the best value for $\beta, \rho, \xi$ and $q_{o}$ and their effects throughout the whole system. Important performance metrics such as throughput, latency, and energy consumption were used to evaluate each parameter adaptation. Experiments were performed using 25 sensor nodes and simulated in 100 seconds. The source node and destination node were set in static mode in all experiments. The number of packets sent per second was defined as the source rate while the number of packets requested per second by destination node was defined as the destination rate. A static amount of bandwidth was supplied during the experiments where Constant Bit Rate (CBR) was used as data traffic. The speed of data transmission among sensor nodes was set to $250 \mathrm{kbps}$. 
Table 1

Simulation Parameters

\begin{tabular}{ll}
\hline \multicolumn{1}{c}{ Parameter } & Value \\
\hline Parameter Adaptation & $\beta, \rho, \xi, q_{o}$ \\
Performance Metric & Throughput, Latency, Energy Consumption \\
Number of Nodes & 25 \\
Source Type, Radius, Rate & Static, Random, 1, 4 \\
Destination Type, Radius, Rate & Static, Random, 1, 0.5 \\
Data Traffic & Constant Bit Rate (CBR) \\
Data Rate & 250 Kbps \\
Simulation Time & 100 seconds \\
Nodes Energy & 50 Joules \\
\hline
\end{tabular}

The first set of experiments evaluated the best value of $\beta$ which was the heuristic value in calculating the probabilistic decision rule to select the potential sensor nodes. Values ranging from 1 to 10 were used in evaluating the routing performance of the ACS. Based on the experimental results, 4 (highlighted in red) is the best value for $\beta$ as presented in Figure 5.1 for throughput, Figure 5.2 for latency, and Figure 5.3 for energy consumption. The optimal $\beta$ value is important to encourage sensor nodes with high capabilities to be selected during the neighbor node searching process.

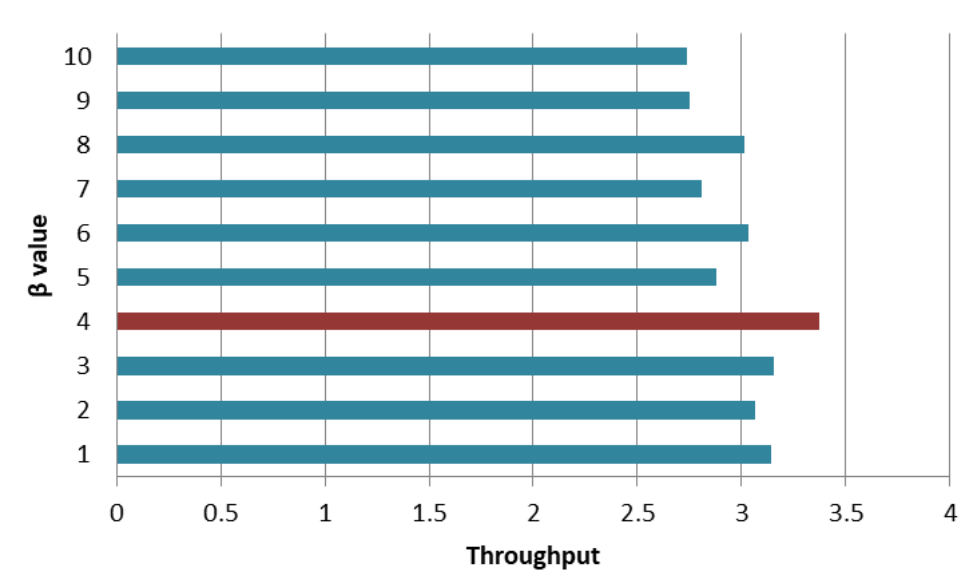

Figure 5.1. Effect of $\beta$ value on throughput of ACS algorithm in WSN. 


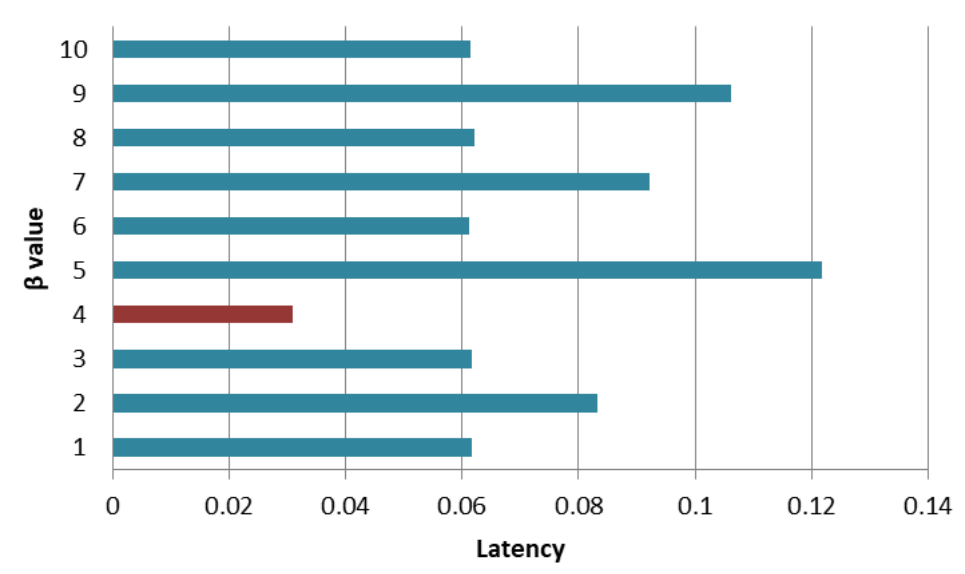

Figure 5.2. Effect of $\beta$ value on latency of ACS algorithm in WSN.

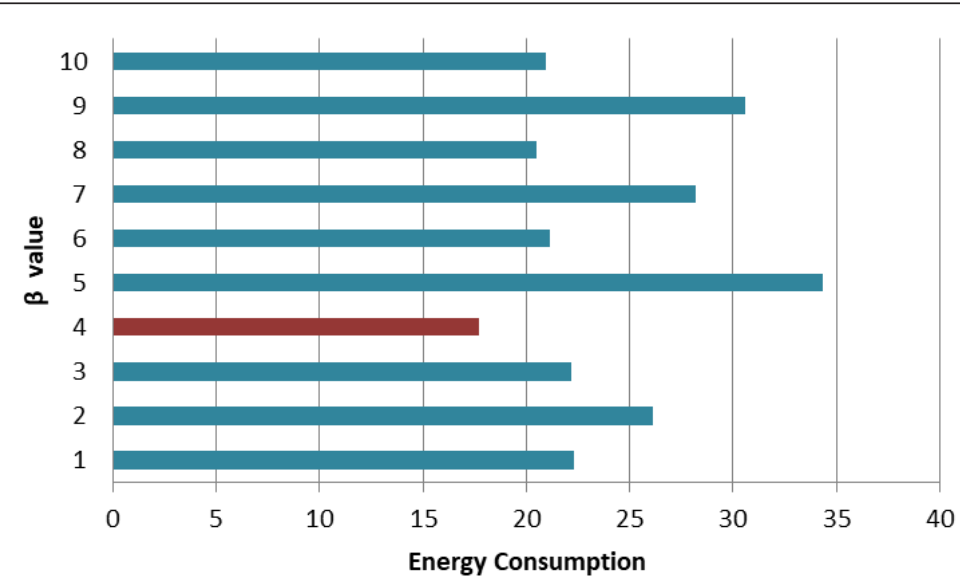

Figure 5.3. Effect of $\beta$ value on energy consumption of ACS algorithm in WSN.

The second set of experiments was undertaken to evaluate the best value of $q_{o}$ to be used as a threshold in the state transition rule, either to explore a new potential path or exploit a previous selected path. The values of $q_{o}$ ranging from 0 to 1 were applied during the experiments to evaluate the optimal threshold that would affect the routing performance of the ACS in the WSN. Based on the experimental results, 0.5 (highlighted in red) is the best value to be used as 
a $q_{o}$ as shown in Figure 5.4 for throughput, Figure 5.5 for latency, and Figure 5.6 for energy consumption. It is important to determine the best value of $q_{o}$ because it affects load balancing among sensor nodes in the system.

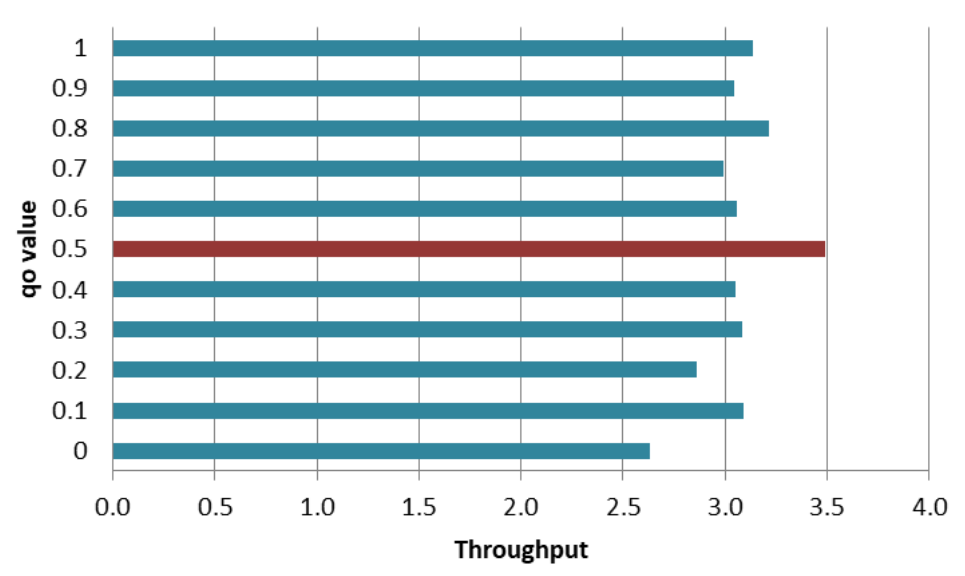

Figure 5.4. Effect of $q_{o}$ value on throughput of ACS algorithm in WSN.

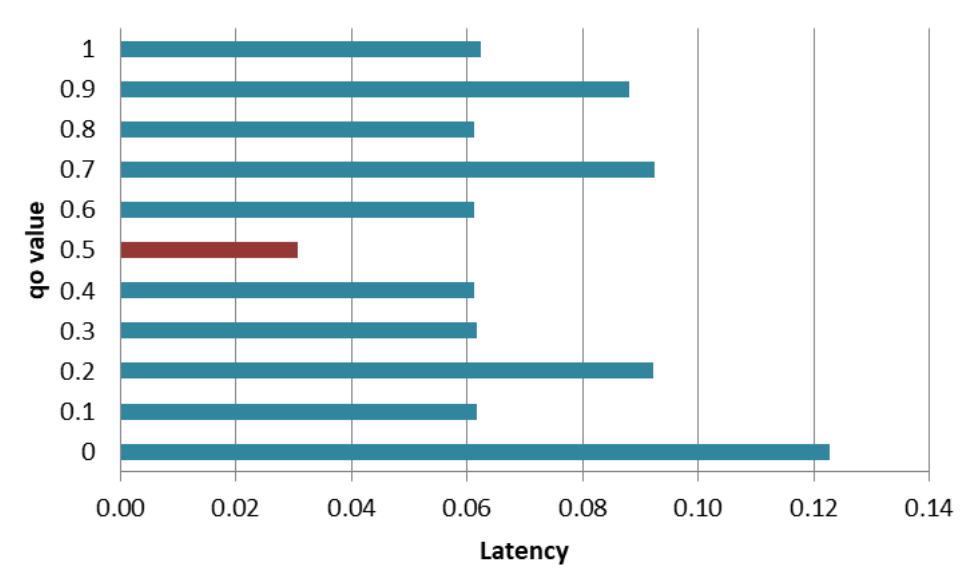

Figure 5.5. Effect of $q_{o}$ value on latency of ACS algorithm in WSN. 


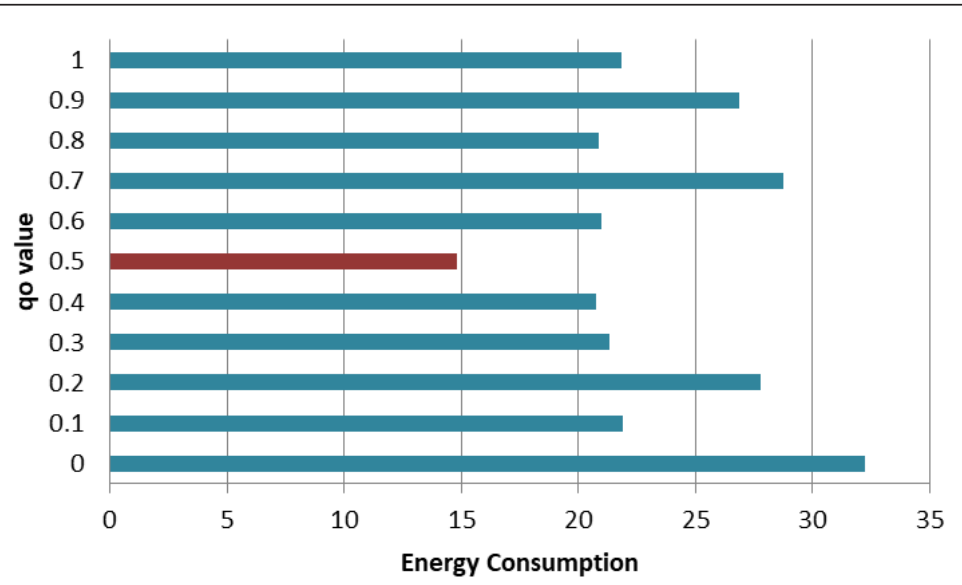

Figure 5.6. Effect of $q_{o}$ value on energy consumption of ACS algorithm in WSN.

The optimal $\xi$ value to be used in calculating the local pheromone update was investigated in the third set of experiments. Local pheromone update encourages new potential path(s) to be selected in balancing the load in the system. Based on the experimental results, 0.3 (highlighted in red) is the optimal value of $\xi$ as displayed in Figure 5.7 for throughput, Figure 5.8 for latency, and Figure 5.9 for energy consumption. These results indicate that $\xi$ value has a huge impact on the number of packets received and the energy efficiency of sensor nodes in the WSN because it controls the reduction of pheromone value that can encourage the exploration of other available sensor nodes and reduces the possibility of hotspot problems.

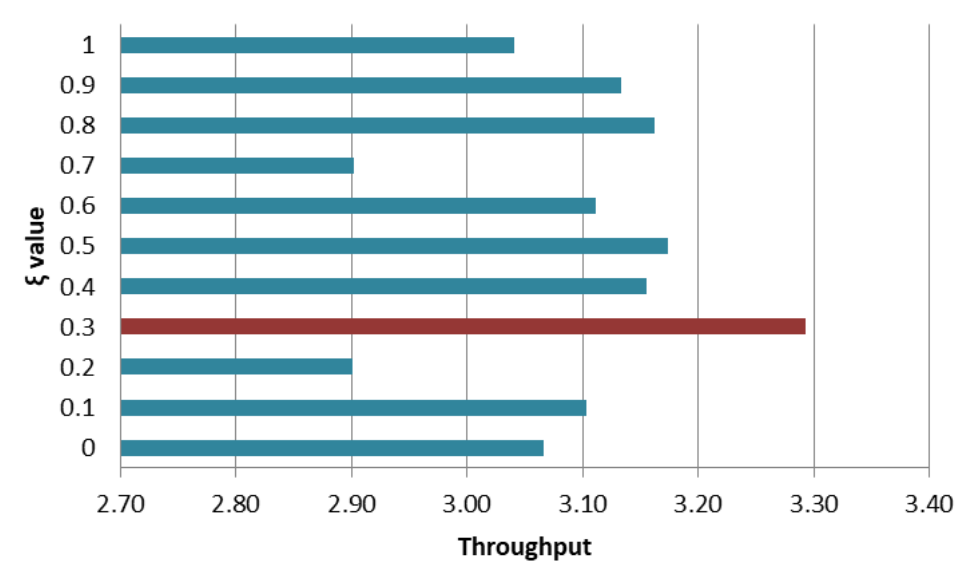

Figure 5.7. Effect of $\xi$ value on throughput of ACS algorithm in WSN. 


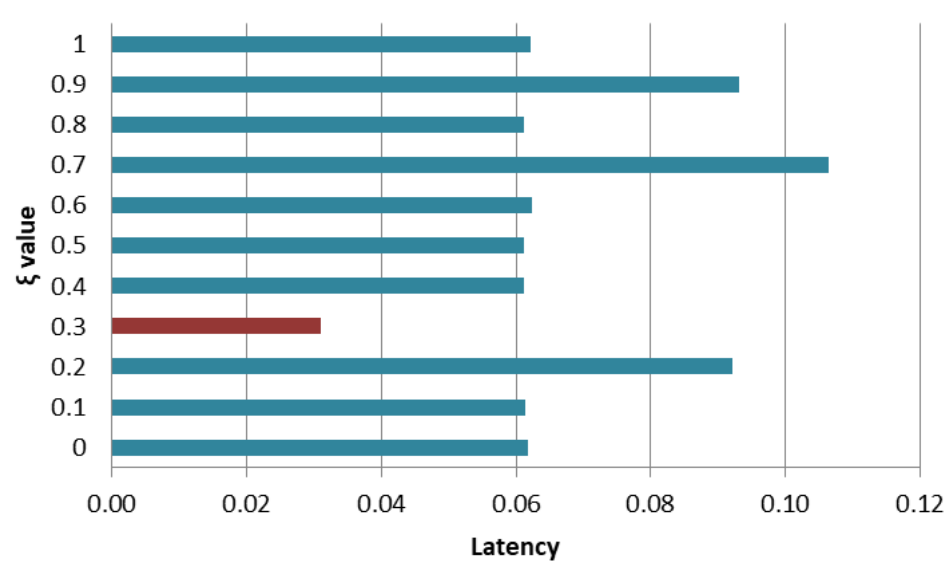

Figure 5.8. Effect of $\xi$ value on latency of ACS algorithm in WSN.

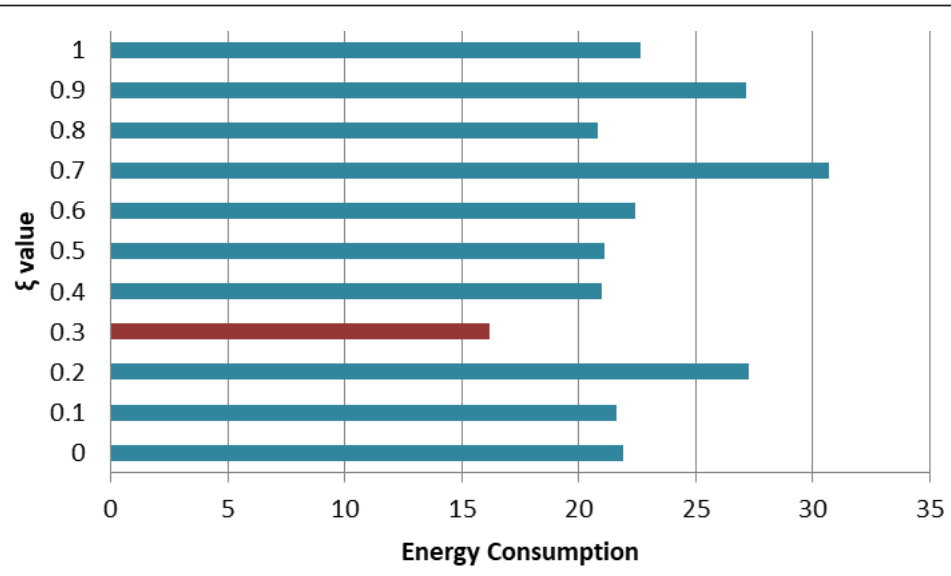

Figure 5.9. Effect of $\xi$ value on energy consumption of ACS algorithm in WSN.

The $\rho$ value, which is the main element in the global pheromone update, will encourage the ant in the next iteration to use the previously optimal routing path. The fourth set of experiments was conducted to investigate the optimal value of $\rho$ which is $0<\rho<1$ to be used by the ACS in the WSN. Based on the experimental results, 0.2 (highlighted in red) is the optimal value of $\rho$ as shown in Figure 5.10 for throughput, Figure 5.11 for latency and Figure 5.12 for energy consumption. These results indicate that the optimal $\rho$ value will 
reduce the number of dead nodes and thus improve the network lifetime of the WSN due to the low energy consumption among sensor nodes.
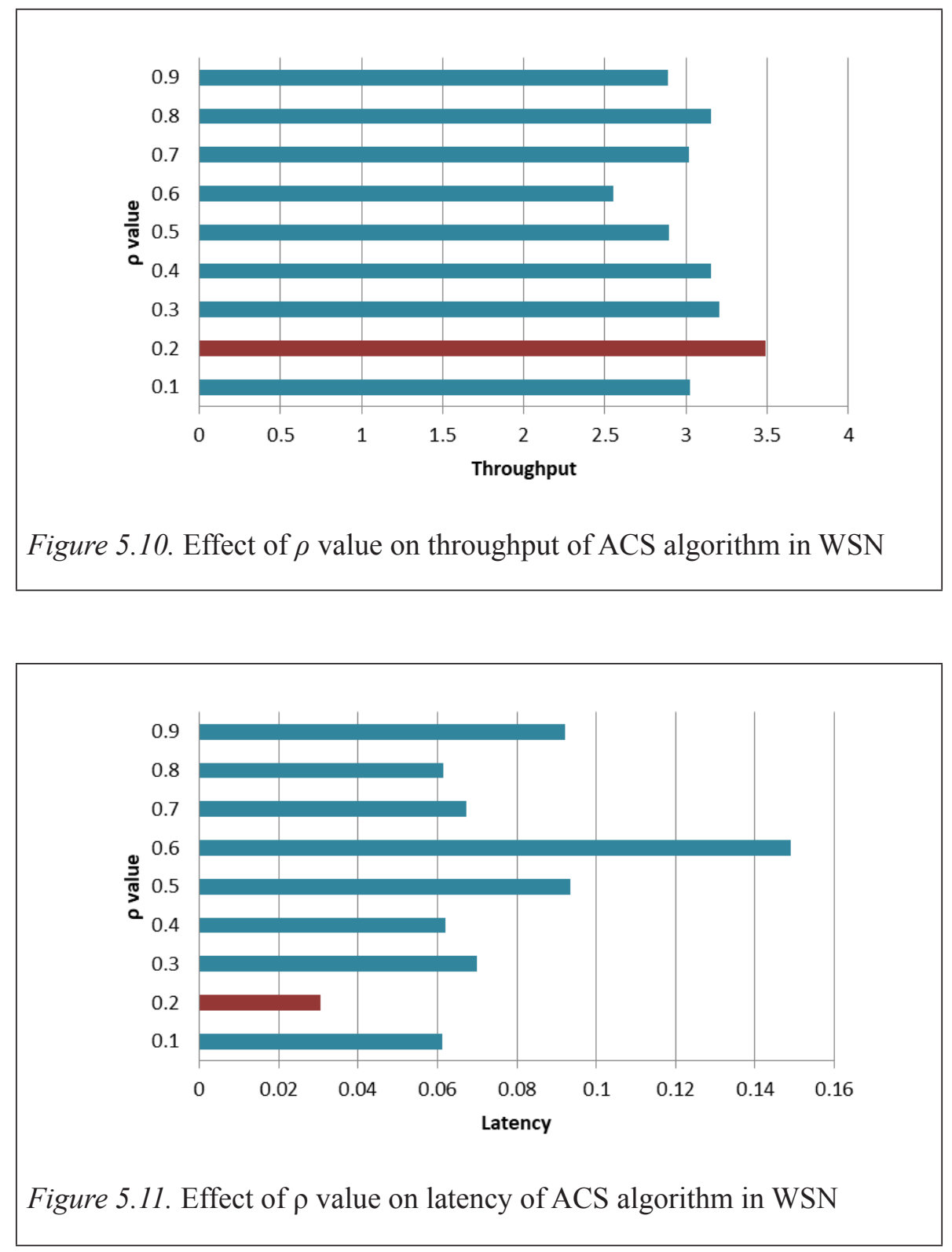

In total, 12 sets of experiments were conducted and results for the optimal values for $\beta, \rho$, $\xi$, and $q_{o}$ for ACS are as listed in Table 2. The optimal $\beta$ value, which is the heuristic value to be used in pseudorandom proportional rule and best sensor nodes with high energy and pheromone value. Both probabilistic 


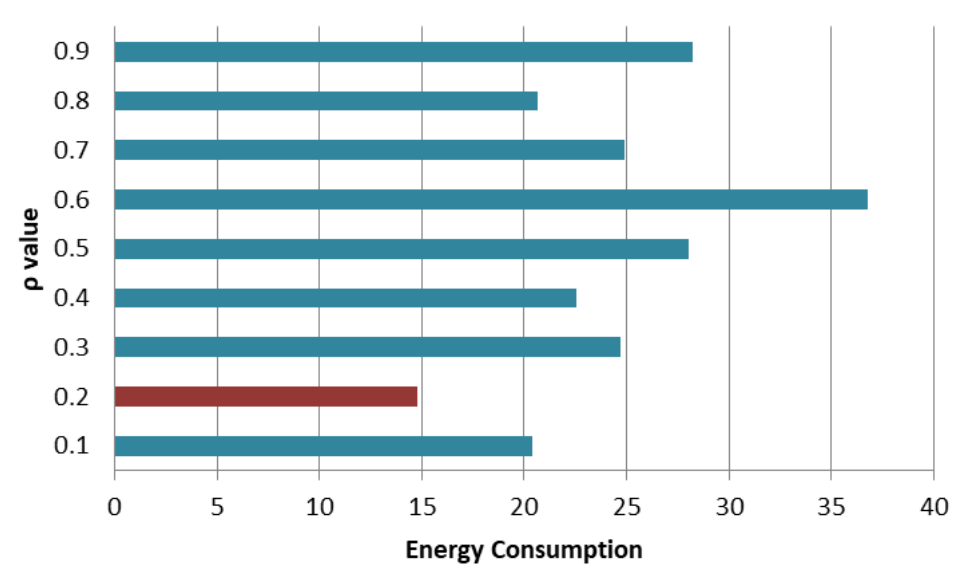

Figure 5.12. Effect of $\rho$ value on energy consumption of EACS(TS) algorithm

decision rule, is 4 as it can influence the selection of the approaches are important in maximizing throughput during packet submission. Experiments were also undertaken to evaluate the best value for $q_{o}$ in controlling the movement of the ant either to explore new potential sensor nodes or to exploit previous optimal sensor nodes. The best value for $q_{o}$ is 0.5 which denotes a $50 \%$ possibility for both exploration and exploitation in ensuring load balancing in the WSN system.

Table 2

Optimal Values for all Parameters

\begin{tabular}{ll}
\hline Parameter & Value \\
\hline$\beta$ & 4 \\
$q_{o}$ & 0.5 \\
$\xi$ & 0.3 \\
$\rho$ & 0.2 \\
\hline
\end{tabular}

The $\xi$ value that is applied in the local pheromone update was also evaluated by a set of experiments. As indicated in Table 2, the best value for $\xi$ is 0.3 where it can help in reducing the pheromone value on visited sensor nodes. This approach can help the ACS in preventing hotspot problems where certain sensor nodes with a high pheromone value will lead to stagnation problems. 
In addition, experiments were completed to determine the best value for $\rho$ to be used in the global pheromone update. The results indicate that the optimal $\rho$ value is 0.2 where it can help optimal sensor nodes to be selected again by any ant in the next iteration. This approach may reduce latency and energy consumption during the search process of sensor nodes to route packets from source node to destination node.

All these parameters are considered as optimal only for the ACS algorithm in a WSN. However, different factors such as type of simulation environment, type of topology, type of packet, and sensor node characteristics may affect optimal performance.

\section{CONCLUSION}

It is undeniably crucial to use the best values for the parameters in optimizing the performance of the ACS algorithm in a WSN. Optimized performance would ensure that the system can operate efficiently to meet its objective as the system can run with minimal routing failure and less energy consumption, higher throughput, and minimal time required to transmit packets from source to destination node. Future work could focus on parameter tuning for other variants of ACO algorithms, under different environments, topologies, and application domains.

\section{ACKNOWLEDGEMENT}

The study was funded by the Transdisciplinary Research Grant Scheme (S/O code 13164), Ministry of Higher Education Malaysia.

\section{REFERENCES}

Al-Behadili, H. N. K., Ku-Mahamud, K. R., \& Sagban, R. (2018). Rule pruning techniques in the ant-miner classification algorithm and its variants: A review. In 2018 IEEE Symposium on Computer Applications \& Industrial Electronics (ISCAIE) (pp. 78-84). IEEE.

Blum, C. (2005). Ant colony optimization: Introduction and recent trends. Physics of Life Reviews, 2(4), 353-373.

Bouarafa, S., Saadane, R., \& Rahmani, M. D. (2018). Inspired from Ants colony: Smart routing algorithm of wireless sensor network. Information, 9(1), 23. https://doi.org/10.3390/info9010023 
Brezina Jr, I., \& Čičková, Z. (2011). Solving the travelling salesman problem using the ant colony optimization. Management Information Systems, 6(4), 10-14.

Bukhari, S., Ku-Mahamud, K. R., \& Morino, H. (2017). Dynamic ACO-based fault tolerance in grid computing. International Journal of Grid and Distributed Computing, 10(12), 117-124.

Camilo, T., Carreto, C., Silva J. S., \& Boavida, F. (2006). An energy-efficient ant-based routing algorithm for wireless sensor networks. In M. Dorigo, L. M. Gambardella, M. Birattari, A. Martiloni, R. Poli, \& T. Stützle (Eds.), Ant Colony Optimization and Swarm Intelligence (pp. 49-59). Springer, Berlin, Heidelberg.

Cecílio, J., \& Furtado, P. (2014). Wireless sensor networks: Concepts and components. In Wireless Sensors in Heterogeneous Networked Systems, 5-25. Springer, Cham.

Dorigo, M., \& Gambardella, L. (1997a). Ant colonies for the travelling salesman problem. BioSystems, 43(2), 73-81.

Dorigo, M., \& Gambardella, L. (1997b). Ant colony system: A cooperative learning approach to the travelling salesman problem. IEEE Transactions on Evolutionary Computation, 1(1), 53-66.

Dorigo, M., \& Stützle, T. (2004). Ant colony optimization. Cambridge, Massachusetts, London, England: MIT Press.

Ferdoush, S., \& Li, X. (2014). Wireless sensor network system design using Raspberry $\mathrm{Pi}$ and Arduino for environmental monitoring applications. Procedia Computer Science, 34, 103-110.

Fidanova, S., \& Marinov, P. (2014). Multi-objective ACO algorithm for WSN layout: Performance according to number of ants. International Journal Metaheuristics, 3(2), 149-161.

Gaertner, D., \& Clark, K. L. (2005). On Optimal Parameters for Ant Colony Optimization Algorithms. International Conference on Artificial Intelligence, (pp. 83-89).

Gilmour, S., \& Dras, M. (2005). Understanding the pheromone system within ant colony optimization. In Australasian Joint Conference on Artificial Intelligence, (pp. 786-789). Springer, Berlin, Heidelberg.

Ismail, M. N., Shukran, M. A., Isa, M. R. M., Adib, M., \& Zakaria, O. (2018). Establishing a soldier wireless sensor network (WSN) communication for military operation monitoring. International Journal of Informatics and Communication Technology, 7(2), 89-95.

Ku-Mahamud, K. R., Din, A. M., \& Nasir, H. J. A. (2011). Enhancement of ant colony optimization for grid load balancing. European Journal of Scientific Research, 64(1), 42-50. 
Rao, Y. C., \& Rani, S. (2015). Energy efficiency and maximizing network lifetime for WSNs using ACO algorithm. International Journal of Innovative Technology and Exploring Engineering, 5(2), 15-20.

Sandra, P. S., Sandeep, C. M., Nair, V., Vindhuja, M. V., Nair, S. S., \& Raja, M. P. (2017). WSN based industrial parameter monitoring using smartwatch. International Conference on Circuit, Power and Computing Technologies (ICCPCT), 1-6. IEEE.

Skinderowicz, R. (2015). Population-based ant colony optimization for sequential ordering problem. In M. Núñez, N. T. Nguyen, D. Camacho, \& B. Trawiński (Eds.), Computational Collective Intelligence (pp. 99109). Springer, Cham.

Skinderowicz, R. (2017). An improved ant colony system for the sequential ordering problem. Computers \& Operations Research, 86, 1-17.

Stützle, T., López-Ibánez, M., Pellegrini, P., Maur, M., De Oca, M. M., Birattari, M., \& Dorigo, M. (2011). Parameter adaptation in ant colony optimization. In Y. Hamadi, E. Monfroy, \& F. Saubion (Eds.), Autonomous Search (pp. 191-215). Springer, Berlin, Heidelberg.

Tennina, S., Di Renzo, M., Kartsakli, E., Graziosi, F., Lalos, A. S., Antonopoulos, A.,...Alonso, L. (2014). WSN4QoL: A WSN-oriented healthcare system architecture. International Journal of Distributed Sensor Networks, 10(5), 1-16.

Wong, K. Y. (2008). Parameter tuning for ant colony optimization: A review. International Conference on Computer and Communication Engineering, (pp. 542-545). IEEE. 